\title{
Electron microscopy of Chaetomium pom 152 shows the assembly of ten-bead string
}

Qi Hao ${ }^{1,3}$, Boyue Zhang ${ }^{2}$, Kangning Yuan ${ }^{2}$, Hang Shi $^{1,2}$ and Günter Blobel ${ }^{1}$

\section{Dear Editor,}

A belt around the waistline of pore membrane (pom) of the nuclear envelope has been detected with electron microscopy ${ }^{1}$. However, its molecular identity, architecture, and function remained unclear. Of the three distinct integral membrane proteins populating the nuclear pore membrane, only pom152 (of yeasts) or gp210 (of multi-cellular organisms) contain a sufficiently large 'trans' domain (over $100 \mathrm{kDa}$ ) that could form such a belt (Fig. 1a and Supplementary Fig. S1).

Using the Phyre2 program ${ }^{2}$ to predict tertiary structures of yeast pom152 and its multi-cellular ortholog, gp210, we first identified ten and fifteen, respectively, immunoglobulin-like (Ig-like) folds which extended over much of these proteins' large trans domain (Fig. 1a, Supplementary Figs. S2 and S3). We next expressed fulllength (FL) pom152 (Ctpom152 $2^{\mathrm{FL}}$ ) of the thermophilic yeast Chaetomium thermophilum in insect cells and purified Ctpom $152^{\mathrm{FL}}$ with detergent extract. Incubation of purified Ctpom $152^{\mathrm{FL}}$ with chymotrypsin revealed a large stable fragment (Fig. 1b), which we identified by mass spectrometry as Ctpom152 $2^{194-1256}$ (Fig. 1b). This fragment represents most of the Ctpom 152 trans domain, starting $\sim 40$ residues downstream of its transmembrane helix (TM) and having lost 14 of its C-terminal residues (Fig. 1a and Supplementary Fig. S1).

The purified Ctpom $152^{194-1256}$ (detergent-free) was examined by negative-stain EM using uranyl acetate. The electron micrographs showed beaded strings of $37 \pm 4 \mathrm{~nm}$,

\footnotetext{
Correspondence: Hang Shi (hangshi@tsinghua.edu.cn)

'Laboratory of Cell Biology, Howard Hughes Medical Institute, The Rockefeller University, 1230 York Ave., New York, NY 10065, USA

${ }^{2}$ Beijing Advanced Innovation Center for Structural Biology, School of Life Sciences, Tsinghua University, Beijing 100084, China

Full list of author information is available at the end of the article.
}

with each bead measuring $\sim 4 \mathrm{~nm}$ in length and $\sim 2 \mathrm{~nm}$ in width (Fig. 1c) with up to ten beads per string, consistent with our structural predictions. On the grid, the beaded strings assumed various shapes, indicating a large degree of flexibility. To further improve the purity and quality, we expressed a new construct Ctpom $152^{186-1270}$ in insect cells. Ctpom $152^{186-1270}$ lacks the upstream transmembrane segment and thus does not require detergent for extraction and purification (Supplementary Information, Fig. 1d). The negative-stain EM of Ctpom $152^{186-1270}$ also showed a beaded string structure (Fig. 1e) indistinguishable from that obtained for the chymotryptic fragment (Ctpom152 $2^{194-1256}$ ) (Fig. 1c). Size-exclusion chromatography coupled to multi-angle light scattering (SECMALS) of Ctpom $152^{186-1270}$ (at $6 \mathrm{mg} / \mathrm{mL}$ per injection) showed that it behaves as a monomer of $130.3 \pm 9.6 \mathrm{kDa}$ (Fig. 1f), close to its theoretical molar mass of $121.7 \mathrm{kDa}$. Likewise, SEC-MALS measurements carried out at two lower concentrations yielded similar molar masses (129.2 $\pm 11.2 \mathrm{kDa}$ at $2 \mathrm{mg} / \mathrm{mL}$ or $136.5 \pm 8.5 \mathrm{kDa}$ at $4 \mathrm{mg} / \mathrm{mL}$ ).

Our data so far left open the question of whether the trans regions of Ctpom152 could oligomerize into lumenal ring. We used purified $C$ tpom $152^{\mathrm{FL}}$, but removed much of the detergent (Supplementary Fig. S4) before rapidly freezing the sample for cryo-EM. As in the negative-stain images, we saw 44-nm long beaded strings (Fig. 1g, h). Some of these strings displayed sufficient density to unequivocally distinguish ten beads (Fig. 1i). Notably, other upstream elements of FL Ctpom152 were not visible in these cryo-electron micrographs, presumably because of their disordered structures; moreover, we could not detect the clear-cut density differences between the beads (Fig. 1i). We conclude that regions other than the trans domain of Ctpom152 are insufficiently compact to be visible in vitreous ice. 
a

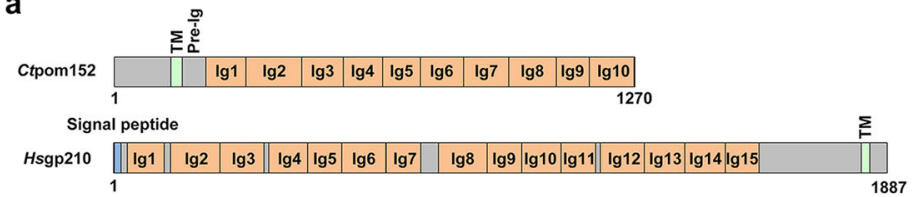

C
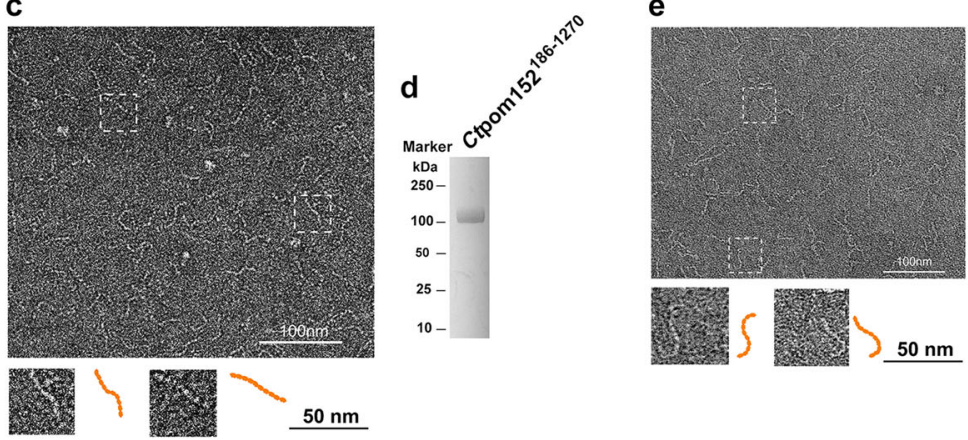

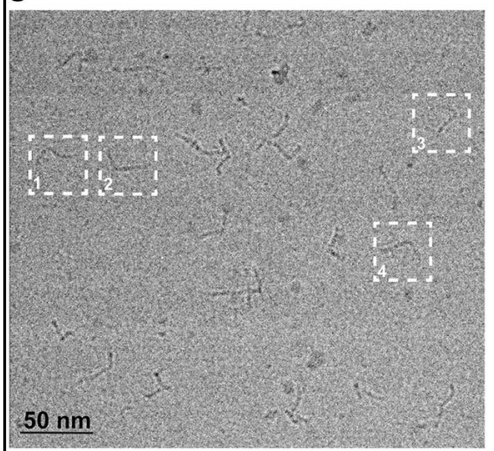

h

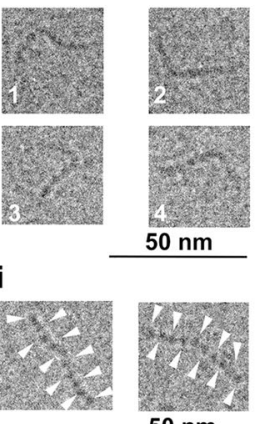

$50 \mathrm{~nm}$
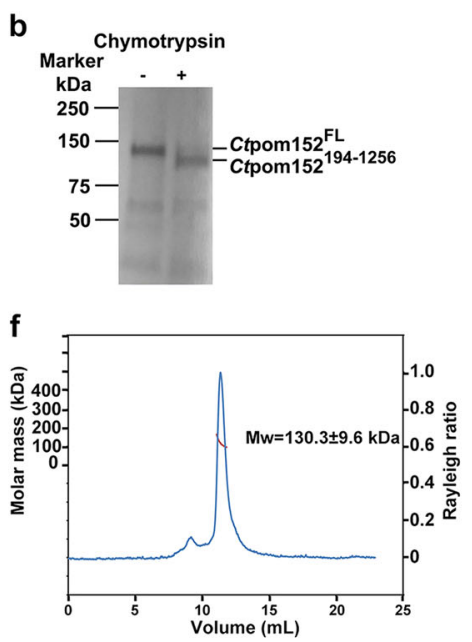

j
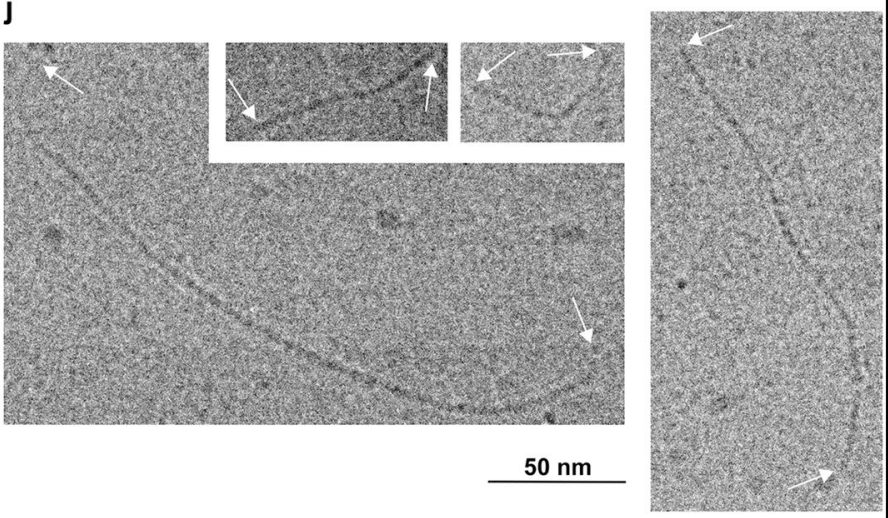

$50 \mathrm{~nm}$

$\mathbf{k}$

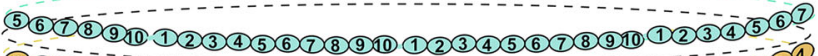
(6) $5(3)(2)$

Fig. 1 Electron microscopy of Ctpom 152 reveals the assembly of beaded strings. a Domain architecture of Ctpom152 and Hsgp210. Regions without a predicted fold are indicated in gray; Ig, immunoglobulin (lg)-like fold; TM, transmembrane segment; pre-lg, the conserved segment between TM and Ig1. See Supplementary Figs. S1, S2 for details. b Limited chymotryptic digestion of full-length (FL) recombinant Ctpom152. c Negative-stain EM of chymotryptic fragment showed beaded and flexible strings of $\sim 40 \mathrm{~nm}$ in length ( $37 \pm 4 \mathrm{~nm}$, number of particles: $N=30$ ); two of the beaded strings (two dashed boxes) are shown enlarged (orange, bottom inserts); up to ten beads are discernible. $\mathbf{d}$ SDS-PAGE and Coomassie Blue staining of purified recombinant Ctpom $152^{186-1270}$. e Like $\mathbf{c}$, negative-stain EM of recombinant Ctpom $152^{186-1270}$ also showed beaded string structures (measures $40 \pm 5 \mathrm{~nm}, N=30$ ). f SEC-MALS indicates that Ctpom152 $186-1270$ is a monomer. $\mathbf{g}$-j Cryo-electron micrograph of Ctpom152 $2^{\mathrm{FL}}$. g Single particle showed flexible beaded strings (measures $44 \pm 3 \mathrm{~nm}, N=30$ ), as seen in negative-stain EM (c, e) and four selected particles (orange with dashed box) are enlarged (h). i Two selected particles with high contrast showing ten beads (indicated by arrow heads) and the large structural variations. $\mathbf{j}$ Ctpom $152^{\mathrm{FL}}$ polymerizes into long continuous strings with no punctuation marks. Particles represent the polymer formed by seven (bottom left), two (top), and five copies (right). $\mathbf{k}$ We speculate that (1) eight trans region of pom152 head-to-tail connect into a flexible ring and (2) two anti-parallel, stacked rings form above (cyan, omitted regions represented by dashed line), below (orange, omitted regions represented by dashed line), and mid-plane (black dashed line)

Strikingly, in up to an estimated $10 \%$ of images (over 2000 images), full-length Ctpom152 molecules appeared as much longer beaded structures (Fig. 1j), measuring in multiples of $44 \mathrm{~nm}$ without consistent punctuation marks between monomers (Fig. 1j). Because the trans segments lacking the $\mathrm{N}$-terminal domain $\left(C\right.$ tpom $152^{186-1270}$ and
Ctpom152 $2^{194-1256}$ ) are unable to oligomerize, these data strongly suggested that the longer beaded structures might arise by head-to-tail oligomerization of the trans domains.

Intriguingly, both pre-Ig and Ig10 are amongst the most conserved regions between pom152 homologs 
(Supplementary Fig. S2), indicative of a common mechanism in yeast. To test it, we mapped the interacting sites between recombinant pre-Ig region and purified Ig10 (Fig. 1a, Supplementary Figs. S2 and S5) from both Chaetomium thermophilum and Saccharomyces cerevisiae. Indeed, both Ctpre-Ig and Scpre-Ig immediately downstream from the TM sufficiently precipitated CtIg10 and $S c \operatorname{Ig} 10$, respectively. The remarkable agreement thus strongly suggests that yeast pom 152 , independent of other poms (pom34 and Ndc1), is capable of assembling into a complete ring.

Taking together, our data here suggest that the large 'trans' domain of Chaetomium thermophilum, Ctpom152, an integral nuclear pore membrane protein, largely consists of ten closely linked Ig folds. Eight head-to-tail connected trans domains would assemble into a continuous ring with diameter of $100 \mathrm{~nm}^{3,4}$. Interestingly, our structural predictions of the presence of fifteen Ig folds in gp210 would yield correspondingly larger rings of $150 \mathrm{~nm}$ in diameter (Fig. $1 \mathrm{k}^{5,6}$ and Supplementary Fig. S3). Because of the symmetry, the 16 trans domains may form 2 eight-member rings, one situated above, the other below mid-plane, collectively representing the lumenal density around the waist of the pore membrane.

Why should NPC require such a structure? As the only conduit between nucleus and cytoplasm, dilation of the central channel to accommodate oversized particles could destabilize the pore membrane. Intriguingly, the trans domain of pom152 (or gp210) bears striking structural similarity to titin, an abundant protein in sarcomeres that fulfills a number of mechanical functions with the most notable one as a passive visco-elastic spring ${ }^{7}$. The existence of an elastic ring in lumen thus could provide the pore membrane with the counter force to maintain its integrity ${ }^{5,8}$.

While preparing our manuscript, a paper by Upla et al. ${ }^{9}$ was published, the results of which were both overlapping and divergent with those of our present paper. Based on $3 \mathrm{D}$ reconstruction of negative-stain EM, Upla et al. ${ }^{9}$ reported that full-length $S$ cpom152 consists of two distinct domains: (1) a large "head" group (comprising of its $375 \mathrm{~N}$-terminal residues) (Fig. S1 and Fig. 4a of Upla et al. $^{9}$ ), and (2) a "lumenal" domain consisting of nine beads in a rigid conformation, with the first one much longer than the remaining eight, all distinctly smaller than the head group (Fig. $6 a$ of Upla et al. ${ }^{9}$ ). In contrast, our EM structures showed ten beads of about equal size in a highly flexible conformation (Fig. 1g, h, i), and the remaining fragment, terminating around the residue 214 (Fig. 1b and Supplementary Figs. S1 and S2), is insufficiently compact to be detected by cryoEM. Moreover, our biochemical analysis suggested that yeast pom152 can oligomerize in a conserved fashion: our predicted "head" (pre-Ig) of one trans domain directly interacts with the "tail" (Ig10) of another, whereby linking multi-trans domains into a single ring (Fig. 1, Supplementary Figs. S2 and S5). Surprisingly, unlike our model, the Scpre-Ig region, Scpom152 $214-265$ was previously interpreted as part of the "head group" (Upla et al. ${ }^{9}$ ), not a constituent of the ring ${ }^{3}$. Better precision in the 'head group' boundary determination, negative stain in the absence of detergent, and higher resolution in 3D reconstruction will be needed in order to resolve the differences between our models.

\section{Acknowledgements}

We thank Alexandra Coutavas and Jianfeng Sun for technical assistance; Joseph P Fernandez and Henrik Molina (Proteomics Resource Center at The Rockefeller University, funded by the Leona M. and Harry B. Helmsley Charitable Trust for mass spectrometer instrumentation) for mass spectrometry analyses; Devrim Acehan, Mark Ebrahim, and Johanna Sotiris (Electron/ Cryoelectron Microscope Resource Centers at The Rockefeller University) for assistance with negative-stain EM and cryoEM; Xiaochun Li and Thomas Walz for discussion; and Bartlomiej Blus and Elias Coutavas for critical reading and editing of the manuscript. This work was funded by Howard Hughes Medical Institute, Rockefeller University (GB) and Beijing Advanced Innovation Center for Structural Biology at Tsinghua University.

\section{Author details}

'Laboratory of Cell Biology, Howard Hughes Medical Institute, The Rockefeller University, 1230 York Ave., New York, NY 10065, USA. ${ }^{2}$ Beijing Advanced Innovation Center for Structural Biology, School of Life Sciences, Tsinghua University, Beijing 100084, China. ${ }^{3}$ Present address: Calico Life Sciences, 1170 Veterans Blvd, South San Francisco, CA 94080, USA

\section{Author contributions}

Q.H., B.Z., H.S., and G.B. designed the study; Q.H., B.Z., K.Y., and H.S. performed the experiments; Q.H., B.Z., H.S., and G.B. analyzed and interpreted the data; Q. H., B.Z., H.S., and G.B. wrote the paper.

Conflict of interest

The authors declare that they have no conflict of interest.

\section{Publisher's note}

Springer Nature remains neutral with regard to jurisdictional claims in published maps and institutional affiliations.

Supplementary Information accompanies the paper at (https://doi.org/ 10.1038/s41421-018-0057-7).

Received: 19 April 2018 Revised: 7 August 2018 Accepted: 8 August 2018 Published online: 18 September 2018

\section{References}

1. Akey, C. W. \& Radermacher, M. Architecture of the xenopus nuclear pore complex revealed by three-dimensional cryo-electron microscopy. J. Cell. Biol. 122, 1-19 (1993)

2. Kelley, L. A., Mezulis, S., Yates, C. M., Wass, M. N. \& Sternberg, M. J. The Phyre2 web portal for protein modeling, prediction and analysis. Nat. Protoc. 10, 845-858 (2015).

3. Kim, S. J. et al. Integrative structure and functional anatomy of a nuclear pore complex. Nature 555, 475-482, https://doi.org/10.1038/nature26003 (2018).

4. Alber, F. et al. The molecular architecture of the nuclear pore complex. Nature 450, 695-701 (2007).

5. Mahamid, J. et al. Visualizing the molecular sociology at the HeLa cell nuclear periphery. Science 351, 969-972 (2016). 
6. Loschberger, A. et al. Super-resolution imaging visualizes the eightfold symmetry of gp210 proteins around the nuclear pore complex and resolves the central channel with nanometer resolution. J. Cell Sci. 125, 570-575 (2012).

7. Herzog, J. A., Leonard, T. R., Jinha, A. \& Herzog, W. Titin (visco-) elasticity in skeletal muscle myofibrils. Mol. \& Cell. Biomech.: MCB 11, 1-17 (2014).
8. Solmaz, S. R., Blobel, G. \& Melcak, I. Ring cycle for dilating and constricting the nuclear pore. Proc. Nat. Acad. Sci. USA 110, 5858-5863 (2013).

9. Upla, P. et al. Molecular Architecture of the Major Membrane Ring Component of the Nuclear Pore Complex. Structure, https://doi.org/10.1016/j.str.2017.01.006 (2017). 